\title{
OPEN Impact of nutritional index on contrast-associated acute kidney injury and mortality after percutaneous coronary intervention
}

\author{
Miyeun Han ${ }^{1,3,4}$, Hye Won Lee ${ }^{2,3}$, Han Cheol Lee ${ }^{2,3}$, Hyo Jin Kim ${ }^{1,3}$, Eun Young Seong ${ }^{1,3}$ \& \\ Sang Heon Song ${ }^{1,3 凶}$
}

The risk of malnutrition in acute kidney injury and mortality in coronary artery disease patients has not been studied. This study aimed to evaluate whether nutritional status assessed by Onodera's prognostic nutritional index (PNI) was related to percutaneous coronary intervention (PCI) outcomes. A total of 3731 patients who received PCI between January 2010 and December 2018 were included. The relationship between $\mathrm{PNI}$ at the time of $\mathrm{PCl}$ and the occurrence of contrast-associated acute kidney injury (AKI) and all-cause death was evaluated using logistic regression and Cox proportional hazards models, respectively. AKI occurred in 271 patients (7.3\%). A low PNI was independently associated with an increased risk of AKI on multivariate logistic regression analysis $(O R 0.96,95 \% \mathrm{CI}$ $0.94-0.98, P=0.001$ ). During the median follow-up of 4.3 years, Kaplan-Meier analysis showed that patients with AKI/low $\mathrm{PNI}<47.8$ had a higher death rate. After adjusting for various risk factors, a low PNI was a significant risk factor for mortality (HR 0.98, Cl 0.96-0.99, $P=0.003)$. A low level of PNI was associated with increased mortality, especially in the group aged over 70 years and female sex. PNI was closely associated with acute kidney outcomes and patient mortality after PCI.

Coronary artery disease is the leading cause of morbidity and premature mortality worldwide ${ }^{1}$. Percutaneous coronary intervention (PCI) is a non-surgical revascularization procedure. PCI relieves symptoms of ischemia and offers survival benefits in ischemic heart disease patients ${ }^{2}$. However, the administration of contrast media during angioplasty can result in contrast-associated acute kidney injury (CA-AKI). This complication is associated with increased intra-hospital stay, long-term decline in kidney function, and increased mortality ${ }^{3,4}$. Although there are many differences depending on the study, the incidence of CA-AKI can occur in as much as $1-50 \%, 5$. Several risk factors, such as preexisting chronic kidney disease (CKD), old age, hemodynamic instability, osmolality and volume of contrast media, have been established, and several risk stratification models to predict CA-AKI using various factors have been developed in previous studies ${ }^{6,7}$.

Malnutrition is highly prevalent among AKI patients ${ }^{8,9}$, and poor nutritional status could increase the incidence of $\mathrm{AKI}^{10}$. Nutrition is associated with immune dysfunction and inflammatory processes ${ }^{10,11}$. AKI, known to be systemic inflammation causing immune cell dysfunction and altered cytokine homeostasis ${ }^{11}$, is also affected by malnutrition. Furthermore, nutrition and diet play an important role in preventing and progressing cardiovascular disease ${ }^{12}$, and malnutrition is a poor prognostic factor for heart disease. However, the effect of undernutrition on CA-AKI and outcome after PCI has not been actively studied.

There are various nutritional assessment tools. The prognostic nutritional index (PNI), calculated from one's serum albumin concentration and total lymphocyte count in the peripheral blood, was developed by Onoder ${ }^{13}$ to determine the nutritional and inflammatory status of surgical patients. PNI is a simple indicator used not only in postoperative outcomes in patients but also in evaluating the nutritional status of patients with malignancies ${ }^{14,15}$,

\footnotetext{
${ }^{1}$ Division of Nephrology, Department of Internal Medicine, Pusan National University Hospital, Busan, Korea. ${ }^{2}$ Division of Cardiology, Department of Internal Medicine, Pusan National University Hospital, Busan, Korea. ${ }^{3}$ Biomedical Research Institute, Pusan National University Hospital, Busan, Korea. ${ }^{4}$ Present address: Division of Nephrology, Department of Internal Medicine, Hallym University Hangang Sacred Heart Hospital, Seoul, Korea. ${ }^{\bowtie}$ email: shsong0209@gmail.com
} 


\begin{tabular}{|c|c|c|c|c|}
\hline Variables & Total $(n=3731)$ & No AKI $(n=3460)$ & AKI $(n=271)$ & $p$ value \\
\hline Age, years & $65.4 \pm 11.3$ & $65.1 \pm 11.2$ & $69.0 \pm 10.8$ & $<0.001$ \\
\hline Sex (female), n (\%) & $1060(28.4)$ & $964(27.9)$ & $96(35.4)$ & 0.010 \\
\hline Diabetes mellitus, n (\%) & $1061(28.4)$ & $935(27.0)$ & $126(46.5)$ & $<0.001$ \\
\hline Hypertension, n (\%) & $1610(43.2)$ & $1459(42.2)$ & $151(55.7)$ & $<0.001$ \\
\hline Current smoker, n (\%) & $1212(32.5)$ & $1132(32.8)$ & $80(29.6)$ & 0.322 \\
\hline Systolic BP, mmHg & $127.6 \pm 20.4$ & $127.5 \pm 19.9$ & $129.0 \pm 25.3$ & 0.348 \\
\hline Diastolic BP, mmHg & $76.2 \pm 13.2$ & $76.1 \pm 13.0$ & $77.1 \pm 15.8$ & 0.328 \\
\hline Body mass index, $\mathrm{kg} / \mathrm{m}^{2}$ & $24.3 \pm 3.2$ & $24.3 \pm 3.2$ & $23.7 \pm 3.6$ & 0.014 \\
\hline Hemoglobin, g/dL & $13.0 \pm 2.0$ & $13.1 \pm 1.9$ & $11.9 \pm 2.3$ & $<0.001$ \\
\hline RDW-CV, \% & $13.3 \pm 1.3$ & $13.3 \pm 1.3$ & $13.7 \pm 1.3$ & $<0.001$ \\
\hline Total lymphocytes, count/ $\mu \mathrm{L}$ & $2049.4 \pm 1009.6$ & $2066.8 \pm 992.4$ & $1827.0 \pm 1188.0$ & 0.001 \\
\hline Albumin, g/dL & $4.2 \pm 0.50$ & $4.2 \pm 0.5$ & $3.8 \pm 0.6$ & $<0.001$ \\
\hline Creatinine, $\mathrm{mg} / \mathrm{dL}$ & $1.01 \pm 0.63$ & $0.97 \pm 0.55$ & $1.62 \pm 1.14$ & $<0.001$ \\
\hline $\mathrm{eGFR}, \mathrm{mL} / \mathrm{min} / 1.73 \mathrm{~m}^{2}$ & $79.5 \pm 23.2$ & $81.4 \pm 21.2$ & $56.5 \pm 33.2$ & $<0.001$ \\
\hline Total bilirubin, mg/dL & $0.6 \pm 0.5$ & $0.7 \pm 0.5$ & $0.6 \pm 0.4$ & 0.168 \\
\hline Uric acid (mg/dL) & $5.8 \pm 1.9$ & $5.7 \pm 1.8$ & $6.7 \pm 2.4$ & $<0.001$ \\
\hline $\mathrm{CRP}(\mathrm{mg} / \mathrm{dL})$ & $0.96 \pm 2.62$ & $0.89 \pm 2.50$ & $1.89 \pm 3.70$ & $<0.001$ \\
\hline Proteinuria, n (\%) & $538(17.5)$ & $413(14.6)$ & $125(53.0)$ & $<0.001$ \\
\hline PNI & $52.1 \pm 7.8$ & $52.5 \pm 7.5$ & $46.6 \pm 9.2$ & $<0.001$ \\
\hline Contrast volume (ml) & $243.4 \pm 93.1$ & $242.1 \pm 90.9$ & $260.9 \pm 116.2$ & 0.010 \\
\hline
\end{tabular}

Table 1. Clinical characteristics of subjects between no AKI and AKI. $A K I$ acute kidney injury, $B P$ blood pressure, $R D W-C V$ red cell distribution width coefficient of variation, $e G F R$ estimated glomerular filtration rate, $C R P C$-reactive protein, $P N I$ prognostic nutritional index.

pulmonary ${ }^{16,17}$, heart ${ }^{18,19}$, and kidney diseases ${ }^{20}$. However, the prognostic value of PNI in CA-AKI and patient mortality after PCI have not yet been validated. We hypothesized that nutritional status was closely related to the occurrence of AKI and patient prognosis after PCI, independent of other well-known risk factors. This study aimed to investigate whether nutritional status assessed by PNI contributed to acute kidney outcomes and patient mortality after PCI.

\section{Results}

Clinical characteristics of subjects with contrast-associated acute kidney injury. The mean age was $65.4 \pm 11.3$ years, and 1060 (28.4\%) were female. A total of 1061 (28.4\%) patients had diabetes mellitus, and 1610 (43.2\%) had hypertension. Acute kidney injury (AKI) occurred in 271 patients (7.3\%). Table 1 shows the clinical characteristics between the no AKI and AKI groups. Patients with AKI were older and had a higher proportion of females, diabetes mellitus, and hypertension. Body mass index was lower in the AKI group. The levels of hemoglobin, albumin, total lymphocyte count, and eGFR were significantly lower in the AKI group. In contrast, the levels of red cell distribution width coefficient of variation (RDW-CV), creatinine, C-reactive protein (CRP), and uric acid were significantly higher in the AKI group. The proportion of patients with proteinuria was higher in the AKI group. The mean PNI level was 52.5 in the no AKI group and 46.6 in the AKI group. The volume of injected contrast media was higher in the AKI group.

Prognostic nutritional index affects the development of contrast associated acute kidney injury. Table 2 lists several factors associated with AKI. On multivariate logistic regression analysis adjusted for age, sex, diabetes mellitus, hypertension, body mass index, hemoglobin, RDW-CV, eGFR, uric acid, CRP, proteinuria, and contrast volume, low PNI was independently associated with an increased risk of AKI (OR 0.96, 95\% CI 0.94-0.98, $P=0.001)$.

We further divided PNI as a categorical variable. The cut-off level was set to 47.8 based on the ROC curve by AKI prediction (Supplementary Fig. 1). The group with PNI $<47.8$ was 1.85 times more susceptible to AKI (Supplementary Table 1) on multiple logistic regression analysis (OR 1.85, 95\% CI 1.31-2.64, P =0.001).

Both prognostic nutritional index and contrast associated acute kidney injury affect patients' survival. The median follow-up duration of the patients was 4.3 years [ $\min 0, \max 9.8]$. Figure 1 shows the Kaplan-Meier curves for patient survival. The subjects with AKI had higher mortality than those in the no AKI group $(P<0.001)$ (Fig. 1A), and the subjects with lower PNI $<47.8$ had higher mortality than those with PNI $\geq 47.8$ group $(P<0.001)$ (Fig. 1B). When we divided the groups according to the presence of AKI and PNI, the group with AKI/ PNI $<47.8$ had the worst survival (Fig. 1C).

In multiple Cox regression analysis adjusted for age, sex, diabetes mellitus, hypertension, smoking, body mass index, hemoglobin, RDW-CV, eGFR, uric acid, CRP, proteinuria, AKI, medication usage at discharge such as 


\begin{tabular}{|l|l|l|l|c|}
\hline \multirow{2}{*}{} & \multicolumn{3}{|l|}{ Univariate analysis } & \multicolumn{2}{l|}{ Multivariable analysis } \\
\cline { 2 - 6 } & OR $[95 \% \mathbf{C I}]$ & $\boldsymbol{p}$ value & OR $[95 \% \mathbf{C I}]$ & $\boldsymbol{p}$ value \\
\hline Age & $1.03[1.02,1.05]$ & $<0.001$ & $1.00[0.98,1.01]$ & 0.800 \\
\hline Sex (female) & $1.42[1.10,1.84]$ & 0.008 & $1.34[0.95,1.89]$ & 0.099 \\
\hline Diabetes mellitus & $2.35[1.83,3.01]$ & $<0.001$ & $1.23[0.89,1.72]$ & 0.215 \\
\hline Hypertension & $1.73[1.35,2.21]$ & $<0.001$ & $1.05[0.76,1.45]$ & 0.772 \\
\hline Current smoker & $0.86[0.66,1.13]$ & 0.290 & - & - \\
\hline Systolic BP & $1.00[1.00,1.01]$ & 0.250 & - & - \\
\hline Diastolic BP & $1.01[1.00,1.02]$ & 0.248 & - & - \\
\hline Body mass index & $0.95[0.91,0.98]$ & 0.006 & $0.99[0.94,1.03]$ & 0.576 \\
\hline Hemoglobin & $0.73[0.69,0.78]$ & $<0.001$ & $0.98[0.89,1.08]$ & 0.707 \\
\hline RDW-CV & $1.17[1.09,1.26]$ & $<0.001$ & $1.01[0.90,1.13]$ & 0.832 \\
\hline Ln (Total lymphocyte) & $0.41[0.32,0.53]$ & $<0.001$ & - & - \\
\hline albumin & $0.22[0.18,0.27]$ & $<0.001$ & - & - \\
\hline eGFR & $0.96[0.96,0.97]$ & $<0.001$ & $0.98[0.97,0.99]$ & $<0.001$ \\
\hline Total bilirubin & $0.75[0.52,1.08]$ & 0.127 & - & - \\
\hline Uric acid & $1.27[1.20,1.35]$ & $<0.001$ & $1.11[1.03,1.19]$ & 0.008 \\
\hline CRP & $1.09[1.06,1.13]$ & $<0.001$ & $0.98[0.94,1.03]$ & 0.451 \\
\hline Proteinuria & $6.60[5.01,8.69]$ & $<0.001$ & $3.24[2.29,4.52]$ & $<0.001$ \\
\hline PNI & $0.91[0.89,0.92]$ & $<0.001$ & $0.96[0.94,0.98]$ & 0.001 \\
\hline Ln (Contrast volume) & $1.45[1.03,2.04]$ & 0.032 & $1.60[1.09,2.36]$ & 0.018 \\
\hline
\end{tabular}

Table 2. Factor related to the development of contrast associated acute kidney injury. OR odds ratio, $C I$ confidence interval, $B P$ blood pressure, $R D W-C V$ red cell distribution width coefficient of variation, $e G F R$ estimated glomerular filtration rate, $C R P$ C-reactive protein, $P N I$ prognostic nutritional index.
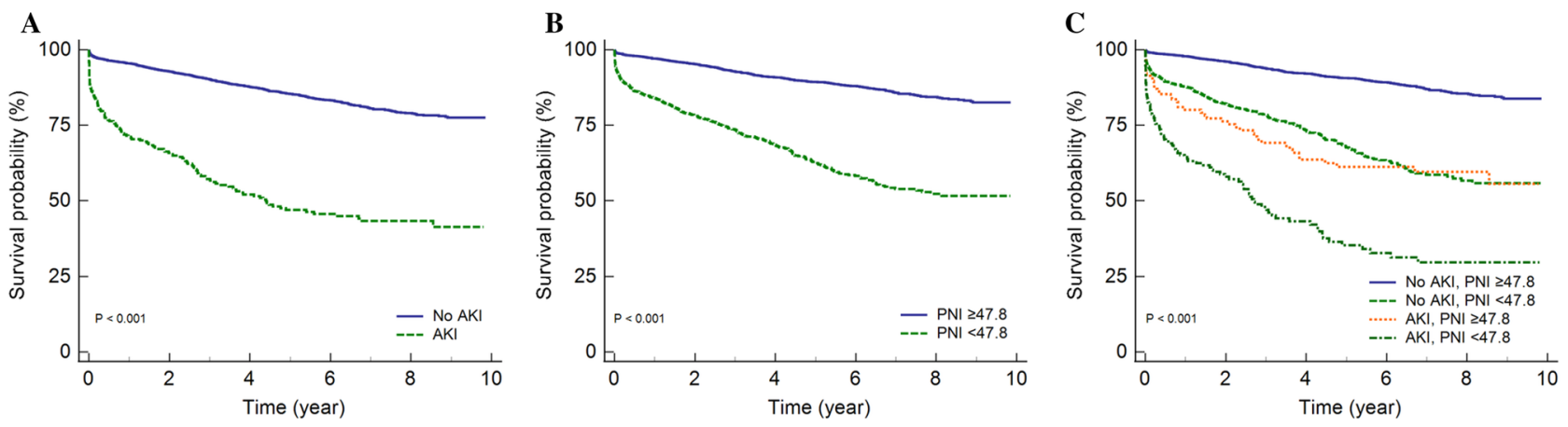

Figure 1. Kaplan-Meier curves for patients' survival. The survival probability of all-cause mortality in patients (A) between patients without AKI and AKI, (B) between $\mathrm{PNI} \geq 47.8$ and $\mathrm{PNI}<47.8$, (C) no AKI/PNI $\geq 47.8$, no $\mathrm{AKI} / \mathrm{PNI}<47.8, \mathrm{AKI} / \mathrm{PNI} \geq 47.8$, and $\mathrm{AKI} / \mathrm{PNI}<47.8$. PNI, prognostic nutritional index; AKI, acute kidney injury.

statin and angiotensin II receptor blocker (ARB)/angiotensin-converting enzyme inhibitor (ACEI), low PNI was a significant risk factor for mortality (HR 0.98, CI 0.96-0.99, $P=0.003$ ) (Table 3). Age, diabetes mellitus, smoking, body mass index, RDW-CV, CRP, proteinuria, AKI, and usage of ARB/ACEI were also significant factors. The results of multiple Cox regression analysis using PNI as a categorical variable are shown in Supplementary Table 2. Further analysis for the cause of death, such as cardiac death or non-cardiac death, is shown in Supplementary Table 3. Low PNI was still a significant risk factor for cardiac death [HR 0.96, CI 0.93-0.98, $P=0.003$ ], and showed similar trend for non-cardiac death [HR 0.98, CI 0.96-1.00, $P=0.075$ ].

When the patients were divided according to the presence of AKI and classified between $\mathrm{PNI}<47.8$ or $\geq 47.8$, the groups with no AKI/PNI $<47.8$ (HR 1.33, CI 1.04-1.69, $P=0.022$ ) as well as AKI/PNI $<47.8$ (HR 2.55, CI $1.77-3.66, P<0.001$ ) had increased risk of mortality compared to the group with no AKI/PNI $\geq 47.8$ (Table 4).

We further analyzed the effects of PNI on various subgroups (Fig. 2). Low PNI levels were associated with increased mortality in the group over 70 years, female sex, no AKI, eGFR $>30 \mathrm{ml} / \mathrm{min} / 1.73 \mathrm{~m}^{2}$, and with or without diabetes. AKI had interaction with PNI $(P$ for interaction $=0.033)$, which means PNI had different effect on mortality according to AKI or no AKI. 


\begin{tabular}{|c|c|c|c|c|}
\hline & \multicolumn{2}{|c|}{ Univariate analysis } & \multicolumn{2}{|c|}{ Multivariable analysis } \\
\hline & HR $[95 \% \mathrm{CI}]$ & $p$ value & HR $[95 \% \mathrm{CI}]$ & $p$ value \\
\hline Age & $1.08[1.07,1.09]$ & $<0.001$ & $1.07[1.06,1.09]$ & $<0.001$ \\
\hline Sex (female) & $1.28[1.09,1.51]$ & 0.003 & $0.85[0.68,1.06]$ & 0.137 \\
\hline Diabetes mellitus & $1.75[1.49,2.05]$ & $<0.001$ & $1.52[1.22,1.88]$ & $<0.001$ \\
\hline Hypertension & $1.27[1.09,1.49]$ & 0.002 & $0.88[0.72,1.08]$ & 0.228 \\
\hline Current smoker & $0.77[0.65,0.92]$ & 0.003 & $1.27[1.00,1.60]$ & 0.047 \\
\hline Systolic BP & $1.00[0.99,1.00]$ & 0.367 & - & - \\
\hline Diastolic BP & $1.00[0.99,1.00]$ & 0.076 & - & - \\
\hline Body mass index & $0.85[0.83,0.88]$ & $<0.001$ & $0.93[0.90,0.96]$ & $<0.001$ \\
\hline Hemoglobin & $0.73[0.70,0.76]$ & $<0.001$ & $0.99[0.93,1.05]$ & 0.748 \\
\hline RDW-CV & $1.24[1.20,1.28]$ & $<0.001$ & $1.13[1.07,1.20]$ & $<0.001$ \\
\hline Ln (Total lymphocyte) & $0.39[0.33,0.46]$ & $<0.001$ & - & - \\
\hline Albumin & $0.25[0.22,0.28]$ & $<0.001$ & - & - \\
\hline eGFR & $0.97[0.97,0.97]$ & $<0.001$ & $0.99[0.99,1.00]$ & 0.051 \\
\hline Total bilirubin & $1.16[1.06,1.26]$ & 0.001 & - & - \\
\hline Uric acid & $1.14[1.10,1.19]$ & $<0.001$ & $1.05[1.00,1.11]$ & 0.069 \\
\hline CRP & $1.11[1.10,1.13]$ & $<0.001$ & $1.05[1.02,1.08]$ & 0.001 \\
\hline Proteinuria & $3.31[2.76,3.96]$ & $<0.001$ & $1.48[1.15,1.89]$ & 0.002 \\
\hline PNI & $0.90[0.89,0.91]$ & $<0.001$ & $0.98[0.96,0.99]$ & 0.003 \\
\hline AKI & $4.54[3.796,5.49]$ & $<0.001$ & $2.17[1.66,2.84]$ & $<0.001$ \\
\hline Statin at discharge & $0.68[0.54,0.86]$ & 0.001 & $0.79[0.61,1.03]$ & 0.084 \\
\hline $\mathrm{ARB} / \mathrm{ACEI}$ at discharge & $0.61[0.50,0.74]$ & $<0.001$ & $0.65[0.52,0.82]$ & $<0.001$ \\
\hline
\end{tabular}

Table 3. Prognostic factors related to patients' mortality. $H R$ hazard ratio, $C I$ confidence interval, $B P$ blood pressure, $R D W-C V$ red cell distribution width coefficient of variation, $e G F R$ estimated glomerular filtration rate, $C R P$ C-reactive protein, $P N I$ prognostic nutritional index, $A K I$ acute kidney injury, $A R B$ angiotensin II receptor blocker, $A C E I$ angiotensin converting enzyme inhibitor.

\begin{tabular}{|l|l|c|}
\hline \multirow{2}{*}{} & Multivariable analysis & \\
\cline { 2 - 3 } & HR $[\mathbf{9 5} \% \mathbf{C I}]$ & $\boldsymbol{p}$ value \\
\hline No AKI/PNI $\geq 47.8$ & Reference & - \\
\hline No AKI/PNI $<47.8$ & $1.33[1.04,1.69]$ & 0.022 \\
\hline AKI/PNI $\geq 47.8$ & $2.76[1.87,4.07]$ & $<0.001$ \\
\hline AKI $/$ PNI $<47.8$ & $2.55[1.77,3.69]$ & $<0.001$ \\
\hline
\end{tabular}

Table 4. Cox regression analysis for mortality groups by AKI/PNI. a Adjusted with age, sex, diabetes mellitus, hypertension, smoking, body mass index, hemoglobin, RDW-CV, eGFR, uric acid, CRP, proteinuria, AKI, medication usage at discharge such as statin and angiotensin II receptor blocker (ARB)/angiotensin converting enzyme inhibitor (ACEI). HR hazard ratio, CI confidence interval, AKI acute kidney injury, PNI prognostic nutritional index.

\section{Discussion}

$\mathrm{PNI}$, as a clinical nutritional index, was closely associated with acute kidney outcome and patient mortality after PCI. Low PNI increases the risk of CA-AKI and mortality after PCI. Patients with CA-AKI and low PNI had the highest mortality rates. Low PNI increases mortality, especially in patients over 70 years or female, and is particularly related to cardiac death. Kidney dysfunction and cardiovascular disease are interconnected and can lead to long-term outcomes, such as mortality. The present study was the first to identify the impact of nutrition on CA-AKI and mortality after PCI.

We defined CA-AKI according to the KDIGO guidelines ${ }^{8}$. Traditionally, contrast-induced AKI was defined as a rise in $\mathrm{SCr}$ of $\geq 0.5 \mathrm{mg} / \mathrm{dL}(\geq 44 \mu \mathrm{mol} / \mathrm{L})$ or a $25 \%$ increase from the baseline value occurring two to five days after contrast exposure. However, this definition was limited in that it missed minor increases in creatinine that correlated with adverse outcomes ${ }^{6}$. Furthermore, other factors, such as medication or hypotension, may precipitate AKI after exposure to contrast media, so the term 'CA-AKI' is preferred ${ }^{7}$. The incidence of CA-AKI after PCI is varied due to different definitions of CA-AKI and study population characteristics. The incidence of CA-AKI after PCI in this study was 7.3\%, and 226 (83.4\%) of the cases belonged to the AKI stage I.

We confirmed that a low PNI increased the risk of CA-AKI. Similarly, previous studies have reported the effects of nutrition on AKI. Epidemiology study revealed that patients with NRS-2002 scores $\geq 3$ had 1.8 times higher incidence of AKI in a 5-year retrospective cohort study ${ }^{10}$. Experimental studies demonstrated that several 


\section{HR $(95 \% \mathrm{CI})$}

\section{Age}

$<70$

$>=70$

Sex

Male

Female

AKI

No

Yes

eGFR

$<=30$

$30-60$

$>60$

DM

No

Yes $p$ value for

interaction

0.276

$0.99(0.96,1.01)$

$0.97(0.95,0.99)$

$0.99(0.97,1.00)$

$0.95(0.93,0.98)$

$0.97(0.95,0.98)$

$1.02(0.99,1.06)$

$0.96(0.90,1.01)$

$0.97(0.94,1.00)$

$0.98(0.96,1.00)$

$0.97(0.95,1.00)$

$0.97(0.95,1.00)$

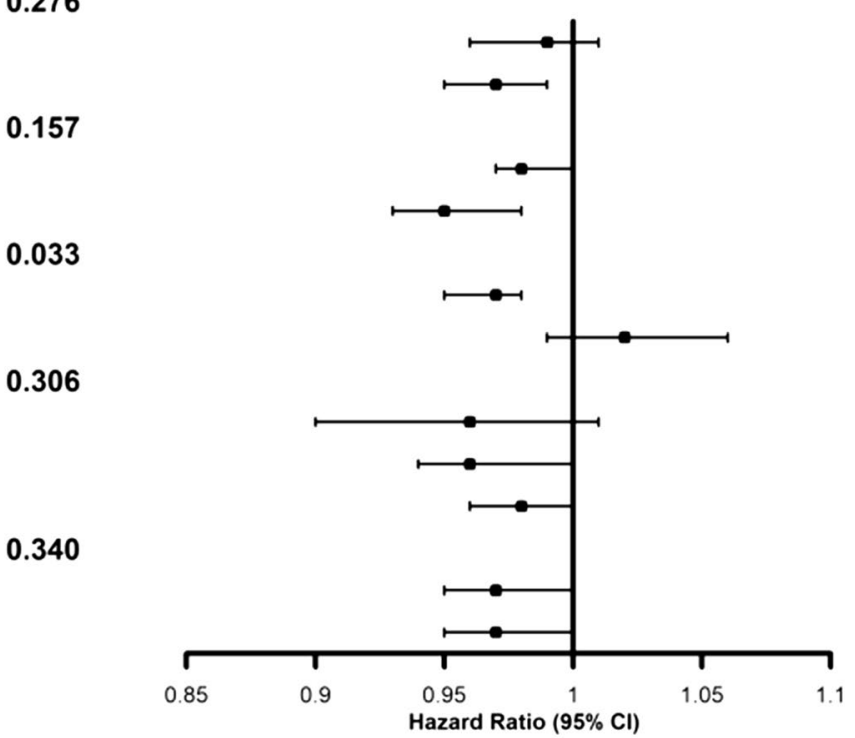

Figure 2. Cox hazard ratio of survival for PNI in various subgroups. A low level of PNI was associated with increased mortality in the group aged over 70 years, female sex, no AKI, and eGFR $>30 \mathrm{ml} / \mathrm{min} / 1.73 \mathrm{~m}^{2}$. PNI, prognostic nutritional index; AKI, acute kidney injury; eGFR, estimated glomerular filtration rate.

nutrients serve as renoprotective agents in AKI. For instance, glutamine administration prevented AKI by downregulating oxidative stress in sepsis-induced AKI rat models ${ }^{21}$. Omega 3 fatty acids decrease polymorphonuclear leukocyte recruitment and cytokine levels in the kidney in ischemic renal injury model ${ }^{22}$. The current study was focused on PNI as a composite nutritional index; PNI is the summation of serum albumin and total lymphocyte counts. Albumin does not only represents the nutritional status ${ }^{23}$ but also acts as an important circulating antioxidant ${ }^{24}$. Albumin acts through multiple binding sites and has free radical-trapping properties. The main mechanisms of contrast-induced nephropathy are vasoconstriction and oxidative stress, followed by renal medullary hypoxia and direct tubular toxicity by contrast media. Thus, a low level of albumin exacerbated CA-AKI. Likewise, lymphocytes can be used as a marker for nutritional status ${ }^{23}$, but various lineages of lymphocytes are also involved in the repair and protection of $\mathrm{AKI}^{25}$.

We also revealed several risk factors for CA-AKI. Preexisting CKD, such as low eGFR and proteinuria, is the strongest risk factor for AKI. Serum uric acid is a risk factor for various heart and kidney diseases and was associated with an increased risk of AKI after coronary angiography ${ }^{12}$. The type and volume of the contrast material affected the incidence of AKI. Current guidelines recommend using iso-osmolar or low-osmolar contrast media with minimized volume (total contrast volume/GFR $<3.7$ ) to prevent $\mathrm{AKI}^{26}$. All contrast media used in our patients were nonionic iso-osmolar or low-osmolarity media. The mean volume of contrast media injected was $243 \mathrm{~mL}$, and $29.9 \%$ of patients received a dose over 3.7 in total contrast volume/GFR. For the high volume of contrast media associated with AKI, we should try to use contrast media cautiously in CKD patients. Diabetes mellitus was not related to the occurrence of AKI.

Various risk factors for mortality after PCI were identified, and AKI showed the highest HR on multiple Cox regression analysis. There is concern that CA-AKI may have been overestimated, affecting the negative effect of performing angiography in patients with $\mathrm{CKD}^{27}$. For instance, Ribitsch et al. showed that the occurrence of contrast-induced AKI did not negatively impact kidney function after three months or mortality after PCI in a prospective cohort ${ }^{28}$. However, Ribitsch's study included only 706 patients with a follow-up period of two years. With a large population and longer follow-up duration, our results clearly showed AKI after PCI as a risk factor for mortality; this is consistent with previous reports ${ }^{29,30}$.

Old age, diabetes mellitus, smoking, and several laboratory values, such as RDW-CV and CRP, were independently associated with increased mortality. CRP, a well-known acute-phase reactant, reflects the degree of underlying inflammation ${ }^{31}$. RDW-CV is a hematological index used to explore the causes of anemia. However, it is now regarded as a prognostic marker that reflects the underlying inflammatory state and is related to patient outcomes ${ }^{32}$. Higher RDW-CV was also a predictor in patients undergoing $\mathrm{PCI}^{33}$ in the previous report as well as $\mathrm{CRP}^{34}$. Our results are in line with those of previous studies, showing that inflammation played a crucial role in the initiation and progression of atherosclerosis, affecting clinical outcomes.

Meanwhile, a low PNI also increases the risk of mortality after PCI. A previous study also reported that PNI was associated with mortality in patients with stable coronary artery disease ${ }^{19}$. Nutritional influences on cardiovascular risk are well demonstrated. Poor quality of diet and undernutrition could aggravate insulin resistance and atherogenesis, promoting proinflammatory process, eventually leading to mortality 35,36 .

PNI did not show discrimination power for mortality in groups with AKI and eGFR $<30 \mathrm{ml} / \mathrm{min} / 1.73 \mathrm{~m}^{2}$. This result could be due to the small population number in AKI $(\mathrm{n}=271)$ groups and eGFR $<30 \mathrm{ml} / \mathrm{min} / 1.73 \mathrm{~m}^{2}$ 
$(\mathrm{n}=154)$. Another study reported that malnutrition in AKI patients was associated with a higher incidence of complications, longer hospitalizations, and higher mortality ${ }^{37}$.

We set the cutoff value of PNI as 47.8, defined by ROC curve using AKI prediction. ROC curve for mortality also set a similar cutoff value [data not shown]. However, we could not define a specific PNI cutoff value. After Onodera suggested the cutoff of PNI value as $45^{13}$, this is frequently used ${ }^{17,38}$. However, the researchers chose several cutoff levels according to study design, such as median, tertile, or ROC analysis ${ }^{15,18,20,39}$. In previous studies, the PNI cut-off value for defining undernutrition varied between 45 and 50. The optimal cut-off value and the classification of the groups by PNI remain unclear.

This study had several limitations. First, this was a single-center retrospective study. Many confounding factors could influence the prognosis of PCI. Second, the PNI was evaluated only once before the PCI. Laboratory changes after PCI were not counted for the analysis. Third, the detection rate of AKI may have been underdiagnosed because of the early discharge of patients. Moreover, we could not suggest AKI to CKD progression or kidney function decline after PCI due to limited follow-up serum creatinine data. We did not consider heart failure; heart failure is one of the important factors for AKI and mortality. In addition, AKI and PNI are highly correlated. To solve the multicollinearity problem, we categorized and integrated AKI/PNI variables. Finally, we did not perform another nutritional assessment, such as subjective global assessment or body composition. However, we analyzed a large number of patients and analyzed long-term patient outcomes using national statistical data related to death.

In conclusion, poor nutritional status, as assessed by PNI, was a risk factor for CA-AKI and all-cause mortality in patients undergoing PCI. This finding suggested that the PNI score, which can be easily determined using serum albumin and lymphocyte counts, was a useful prognostic marker in patients undergoing PCI. Clinicians should be careful when diagnosing and treating patients with coronary artery disease with low PNI.

\section{Methods}

Study population. We retrospectively screened 4,096 patients who underwent PCI at Pusan National University Hospital between January 2010 and December 2018. If the subjects underwent repetitive PCI during the study period, only the first case was included. Patients who already received hemodialysis due to kidney failure $(n=137)$, died within $48 \mathrm{~h}$ after PCI $(n=52)$ or did not have serum creatinine $(n=45)$ or PNI $(n=131)$ values, were excluded. A total of 3,731 subjects were included in the final analysis.

Data sources and measures. The data were retrieved from electronic medical records. The data collected included age, sex, body mass index, history of diabetes, hypertension, blood pressure, and laboratory findings, including serum creatinine, hemoglobin, albumin, and urine dipstick albumin results. Mortality data were obtained from the Microdata Integrated System (MDIS) ${ }^{8}$, generated by Statistics Korea.

CA-AKI was defined as a rise in serum creatinine $\geq 0.3$-or 1.5 than the baseline value within $48 \mathrm{~h}$ after PCI according to KDIGO guidelines ${ }^{40}$. Estimated GFR (eGFR) was calculated through the CKD-EPI equation ${ }^{41}$. Proteinuria was defined as a urine dipstick test result of $\geq 1+{ }^{42}$. PNI was calculated as $10 \times$ serum albumin (g/ $\mathrm{dL})+0.005 \times$ total lymphocyte count $\left(\text { per } \mathrm{mm}^{3}\right)^{13}$.

Statistical analysis. All variables with a normal distribution were expressed as mean \pm standard deviation. Categorical variables were expressed as numbers and proportions, and comparisons were made using the chisquare test. Logistic regression analysis was conducted to analyze the factors associated with AKI. The ability to predict CA-AKI was evaluated using the area under the curve (AUC) in the receiver operating characteristic (ROC) curve. The cumulative survival rates were estimated using the Kaplan-Meier method, and differences between survival curves were compared using the log-rank test. Multivariate Cox regression models for mortality were constructed using the confounding factors. Significant variables in the univariate model were entered into a multivariable model. Among the variables collected, serum albumin levels and lymphocyte counts were not included in the multiple logistic or Cox regression models because they were included in the calculation of PNI. Variables included in all multivariable analyses were tested for multicollinearity. We also examined the association between PNI and mortality through a subgroup analysis using a fully adjusted multivariate Cox regression model. The patients were stratified according to age, sex, presence of diabetes mellitus, baseline eGFR, and CA-AKI. All analyses were performed using the R statistical software package (version 4.0.1; R Development Core Team; R Foundation for Statistical Computing, Vienna, Austria).

Ethical statement. The study was conducted in accordance with the Declaration of Helsinki, and the study was approved by the Institutional Review Board of Pusan National University Hospital (H-1909-014-083). The need for informed consent was waived because of its retrospective design.

Received: 8 January 2021; Accepted: 18 March 2021

Published online: 29 March 2021

\section{References}

1. Collaborators, G. B. D. C. o. D. Global, regional, and national age-sex-specific mortality for 282 causes of death in 195 countries and territories, 1980-2017: a systematic analysis for the Global Burden of Disease Study 2017. Lancet 392, 1736-1788. https://doi. org/10.1016/S0140-6736(18)32203-7 (2018). 
2. Terkelsen, C. J. et al. Primary PCI as the preferred reperfusion therapy in STEMI: it is a matter of time. Heart 95, 362-369. https:// doi.org/10.1136/hrt.2007.139493 (2009).

3. Weisbord, S. D. et al. Associations of increases in serum creatinine with mortality and length of hospital stay after coronary angiography. J. Am. Soc. Nephrol. 17, 2871-2877. https://doi.org/10.1681/ASN.2006030301 (2006).

4. James, M. T. et al. Acute kidney injury following coronary angiography is associated with a long-term decline in kidney function. Kidney Int. 78, 803-809. https://doi.org/10.1038/ki.2010.258 (2010).

5. Han, S. J. \& Lee, H. T. Mechanisms and therapeutic targets of ischemic acute kidney injury. Kidney Res. Clin. Pract. 38, 427-440. https://doi.org/10.23876/j.krcp.19.062 (2019).

6. Rear, R., Bell, R. M. \& Hausenloy, D. J. Contrast-induced nephropathy following angiography and cardiac interventions. Heart 102, 638-648. https://doi.org/10.1136/heartjnl-2014-306962 (2016).

7. Mehran, R., Dangas, G. D. \& Weisbord, S. D. Contrast-associated acute kidney injury. N. Engl. J. Med. 380, 2146-2155. https:// doi.org/10.1056/NEJMra1805256 (2019).

8. Statistics Korea. Microdata integrated service. [cited 2020 Dec 01]. Available from: https://mdis.kostat.go.kr.

9. Meyer, D., Mohan, A., Subev, E., Sarav, M. \& Sturgill, D. Acute kidney injury incidence in hospitalized patients and implications for nutrition support. Nutr. Clin. Pract. 35, 987-1000. https://doi.org/10.1002/ncp.10595 (2020).

10. Li, C. et al. Malnutrition screening and acute kidney injury in hospitalised patients: a retrospective study over a 5-year period from China. Br. J. Nutr. 123, 337-346. https://doi.org/10.1017/S000711451900271X (2020).

11. Singbartl, K., Formeck, C. L. \& Kellum, J. A. Kidney-immune system crosstalk in AKI. Semin. Nephrol. 39, 96-106. https://doi. org/10.1016/j.semnephrol.2018.10.007 (2019).

12. Eilat-Adar, S., Sinai, T., Yosefy, C. \& Henkin, Y. Nutritional recommendations for cardiovascular disease prevention. Nutrients 5, 3646-3683. https://doi.org/10.3390/nu5093646 (2013).

13. Onodera, T., Goseki, N. \& Kosaki, G. Prognostic nutritional index in gastrointestinal surgery of malnourished cancer patients. Nihon Geka Gakkai Zasshi 85, 1001-1005 (1984).

14. Hua, X. et al. The value of Prognostic Nutritional Index (PNI) in predicting survival and guiding radiotherapy of patients with T1-2N1 breast cancer. Front. Oncol. 9, 1562. https://doi.org/10.3389/fonc.2019.01562 (2019).

15. Li, D., Yuan, X., Liu, J., Li, C. \& Li, W. Prognostic value of prognostic nutritional index in lung cancer: a meta-analysis. J. Thorac. Dis. 10, 5298-5307. https://doi.org/10.21037/jtd.2018.08.51 (2018).

16. Tsukahara, T. et al. Factors for predicting outcomes among non-HIV patients with pulmonary tuberculosis. Intern. Med. 56, 3277-3282. https://doi.org/10.2169/internalmedicine.9120-17 (2017).

17. Moon, S. W. et al. Impact of prognostic nutritional index on outcomes in patients with Mycobacterium avium complex pulmonary disease. PLoS ONE 15, e0232714. https://doi.org/10.1371/journal.pone.0232714 (2020).

18. Cheng, Y. L. et al. Prognostic nutritional index and the risk of mortality in patients with acute heart failure. J. Am. Heart Assoc.6, 1. https://doi.org/10.1161/JAHA.116.004876 (2017).

19. Wada, H. et al. Relationship between the prognostic nutritional index and long-term clinical outcomes in patients with stable coronary artery disease. J. Cardiol. 72, 155-161. https://doi.org/10.1016/j.jjcc.2018.01.012 (2018).

20. Zhang, H., Tao, Y., Wang, Z. \& Lu, J. Evaluation of nutritional status and prognostic impact assessed by the prognostic nutritional index in children with chronic kidney disease. Medicine (Baltimore) 98, e16713. https://doi.org/10.1097/MD.0000000000016713 (2019).

21. Hu, Y. M., Pai, M. H., Yeh, C. L., Hou, Y. C. \& Yeh, S. L. Glutamine administration ameliorates sepsis-induced kidney injury by downregulating the high-mobility group box protein-1-mediated pathway in mice. Am. J. Physiol. Renal Physiol. 302, F150-158. https://doi.org/10.1152/ajprenal.00246.2011 (2012).

22. Hassan, I. R. \& Gronert, K. Acute changes in dietary omega-3 and omega- 6 polyunsaturated fatty acids have a pronounced impact on survival following ischemic renal injury and formation of renoprotective docosahexaenoic acid-derived protectin D1. J. Immunol. 182, 3223-3232. https://doi.org/10.4049/jimmunol.0802064 (2009).

23. Bharadwaj, S. et al. Malnutrition: laboratory markers vs nutritional assessment. Gastroenterol. Rep. (Oxf.) 4, 272-280. https://doi. org/10.1093/gastro/gow013 (2016).

24. Roche, M., Rondeau, P., Singh, N. R., Tarnus, E. \& Bourdon, E. The antioxidant properties of serum albumin. FEBS Lett. 582, 1783-1787. https://doi.org/10.1016/j.febslet.2008.04.057 (2008).

25. Weller, S., Varrier, M. \& Ostermann, M. Lymphocyte function in human acute kidney injury. Nephron 137, 287-293. https://doi. org/10.1159/000478538 (2017).

26. Neumann, F. J. et al. 2018 ESC/EACTS guidelines on myocardial revascularization. Eur. Heart. J 40, 87-165. https://doi.org/10. 1093/eurheartj/ehy394 (2019).

27. McDonald, J. S. et al. Acute kidney injury after intravenous versus intra-arterial contrast material administration in a paired cohort. Invest. Radiol. 51, 804-809. https://doi.org/10.1097/RLI.0000000000000298 (2016).

28. Ribitsch, W., Horina, J. H., Quehenberger, F., Rosenkranz, A. R. \& Schilcher, G. Contrast induced acute kidney injury and its impact on mid-term kidney function, cardiovascular events and mortality. Sci. Rep. 9, 16896. https://doi.org/10.1038/s41598-019-53040-5 (2019).

29. Narula, A. et al. Contrast-induced acute kidney injury after primary percutaneous coronary intervention: results from the HORIZONS-AMI substudy. Eur. Heart J. 35, 1533-1540. https://doi.org/10.1093/eurheartj/ehu063 (2014).

30. Kooiman, J. et al. Association between acute kidney injury and in-hospital mortality in patients undergoing percutaneous coronary interventions. Circ. Cardiovasc. Interv. 8, e002212. https://doi.org/10.1161/CIRCINTERVENTIONS.114.002212 (2015).

31. Wilson, P. W. et al. C-reactive protein and reclassification of cardiovascular risk in the Framingham Heart Study. Circ. Cardiovasc. Qual. Outcomes 1, 92-97. https://doi.org/10.1161/CIRCOUTCOMES.108.831198 (2008).

32. Agarwal, S. Red cell distribution width, inflammatory markers and cardiorespiratory fitness: results from the National Health and Nutrition Examination Survey. Indian Heart J. 64, 380-387. https://doi.org/10.1016/j.ihj.2012.06.006 (2012).

33. Bao, D. et al. Prognostic value of red cell distribution width in patients undergoing percutaneous coronary intervention: a metaanalysis. BMJ Open 10, e033378. https://doi.org/10.1136/bmjopen-2019-033378 (2020).

34. Tousoulis, D., Papageorgiou, N. \& Stefanadis, C. Is C-reactive protein a prognostic marker after angioplasty?. Heart 95, 957-959. https://doi.org/10.1136/hrt.2008.156927 (2009).

35. Dalvi, P. S. et al. Long-term metabolic effects of malnutrition: liver steatosis and insulin resistance following early-life protein restriction. PLoS ONE 13, e0199916. https://doi.org/10.1371/journal.pone.0199916 (2018).

36. De Caterina, R., Zampolli, A., Del Turco, S., Madonna, R. \& Massaro, M. Nutritional mechanisms that influence cardiovascular disease. Am. J. Clin. Nutr. 83, 421S-426S. https://doi.org/10.1093/ajcn/83.2.421S (2006).

37. Fiaccadori, E. et al. Prevalence and clinical outcome associated with preexisting malnutrition in acute renal failure: a prospective cohort study. J. Am. Soc. Nephrol. 10, 581-593 (1999).

38. Kanda, M. et al. Nutritional predictors of postoperative outcome in pancreatic cancer. Br. J. Surg. 98, 268-274. https://doi.org/10. 1002/bjs.7305 (2011)

39. Yang, Y. et al. Prognostic significance of preoperative prognostic nutritional index in colorectal cancer: results from a retrospective cohort study and a meta-analysis. Oncotarget 7, 58543-58552. https://doi.org/10.18632/oncotarget.10148 (2016).

40. Kidney Disease: Improving Global Outcomes (KDIGO) Acute Kidney Injury Work Group. KDIGO Clinical Practice Guideline for Acute Kidney Injury. Kidney Int. Suppl. 2, 1-138. https://doi.org/10.1038/kisup.2011.34 (2012). 
41. Levey, A. S. et al. A new equation to estimate glomerular filtration rate. Ann. Intern. Med. 150, 604-612. https://doi.org/10.7326/ 0003-4819-150-9-200905050-00006 (2009).

42. Lim, D. et al. Diagnostic accuracy of urine dipstick for proteinuria in older outpatients. Kidney Res. Clin. Pract. 33, 199-203. https:// doi.org/10.1016/j.krcp.2014.10.003 (2014).

\section{Acknowledgements}

This study was supported by Biomedical Research Institute Grant (2019B035), Pusan National University Hospital. We thank Department of Biostatistics, Biomedical Research Institute, Pusan National University Hospital.

\section{Author contributions}

Study concept and design: M.H. and S.H.S. Acquisition of data: H.W.L. and H.C.L. Analysis and interpretation of data: E.Y.S. Statistical analysis: H.J.K. Drafting of the manuscript: M.H. revision of manuscripts: S.H.S.

\section{Competing interests}

The authors declare no competing interests.

\section{Additional information}

Supplementary Information The online version contains supplementary material available at https://doi.org/ 10.1038/s41598-021-86680-7.

Correspondence and requests for materials should be addressed to S.H.S.

Reprints and permissions information is available at www.nature.com/reprints.

Publisher's note Springer Nature remains neutral with regard to jurisdictional claims in published maps and institutional affiliations.

(c) (i) Open Access This article is licensed under a Creative Commons Attribution 4.0 International License, which permits use, sharing, adaptation, distribution and reproduction in any medium or format, as long as you give appropriate credit to the original author(s) and the source, provide a link to the Creative Commons licence, and indicate if changes were made. The images or other third party material in this article are included in the article's Creative Commons licence, unless indicated otherwise in a credit line to the material. If material is not included in the article's Creative Commons licence and your intended use is not permitted by statutory regulation or exceeds the permitted use, you will need to obtain permission directly from the copyright holder. To view a copy of this licence, visit http://creativecommons.org/licenses/by/4.0/.

(C) The Author(s) 2021 